\title{
ONE-PARAMETER FAMILIES OF CIRCLE DIFFEOMORPHISMS WITH STRICTLY MONOTONE ROTATION NUMBER
}

\author{
KIRAN PARKHE \\ (Communicated by Bryna Kra)
}

\begin{abstract}
We show that if $f: S^{1} \times S^{1} \rightarrow S^{1} \times S^{1}$ is $C^{2}$, with $f(x, t)=$ $\left(f_{t}(x), t\right)$, and the rotation number of $f_{t}$ is equal to $t$ for all $t \in S^{1}$, then $f$ is topologically conjugate to the linear Dehn twist of the torus $\left(\begin{array}{ll}1 & 1 \\ 0 & 1\end{array}\right)$. We prove a differentiability result where the assumption that the rotation number of $f_{t}$ is $t$ is weakened to say that the rotation number is strictly monotone in $t$.
\end{abstract}

\section{INTRODUCTION}

In this article we consider homeomorphisms and diffeomorphisms of the circle $S^{1}=\mathbb{R} / \mathbb{Z}$. They are always assumed to preserve orientation. Following Poincaré, homeomorphisms of the circle may be classified according to their rotation number, which is defined as follows.

Let $f$ be a homeomorphism of the circle, and let $\tilde{f}: \mathbb{R} \rightarrow \mathbb{R}$ be a lift of $f$ (that is, $\pi(\tilde{f}(x))=f(\pi(x))$ for all $x \in \mathbb{R}$, where $\pi$ is the canonical projection). Define the translation number to be $\tau(\tilde{f})=\lim _{n \rightarrow \infty} \frac{\tilde{f}^{n}(0)}{n}$; it is a fact that this limit will always exist. The value of $\tau(\tilde{f})$ does depend on the choice of lift, but for another lift $\tilde{f}^{\prime}$ we will have $\tau(\tilde{f})-\tau\left(\tilde{f}^{\prime}\right) \in \mathbb{Z}$. Therefore, we define the rotation number of $f$ to be $\rho(f)=\pi(\tau(\tilde{f}))$; this does not depend on the choice of lift. We will write rotation numbers as if they were real numbers, understanding that when we write $\rho(f)=x$ we really mean $\rho(f)=x+\mathbb{Z}$.

Poincaré's classification theorem provides strong connections between the rotation number and the dynamics of $f$. It says that $\rho(f)=0$ if and only if $f$ has at least one fixed point (more generally, $\rho(f) \in \mathbb{Q}$ if and only if $f$ has a periodic point) and that $\rho(f) \notin \mathbb{Q}$ if and only if $f$ is topologically semi-conjugate to an irrational rotation. In fact, according to Denjoy's theorem, if $\rho(f) \notin \mathbb{Q}$ and $f$ is a $C^{1}$ diffeomorphism such that the derivative $f^{\prime}$ has bounded variation (in particular, if $f$ is $C^{2}$ ), then $f$ is topologically conjugate to an irrational rotation. Note that a similar statement is false in the case of a rational rotation number (even under strong smoothness assumptions): having a rational rotation number implies that there exists a periodic orbit, not the much stronger statement that every orbit is

Received by the editors December 12, 2011 and, in revised form, February 7, 2012 and February 14, 2012.

2010 Mathematics Subject Classification. Primary 37C15, 37E10, 37E45; Secondary 37C05.

Key words and phrases. Rotation number, strict monotonicity, topological conjugacy. 
periodic with the same period (which is equivalent to being conjugate to a rational rotation). For an excellent discussion of Poincaré's theory, see [2, [3, 7], and for Denjoy's theory, [2], 7].

A rotation number is a function $\rho: \operatorname{Homeo}_{+}\left(S^{1}\right) \rightarrow \mathbb{R} / \mathbb{Z}$, where $\operatorname{Homeo}_{+}\left(S^{1}\right)$ is the group of orientation-preserving homeomorphisms of the circle. Note that the function $\rho$ is not a homomorphism. One can consider the restriction of $\rho$ to the subgroups $\operatorname{Diff}_{+}^{r}\left(S^{1}\right), 1 \leq r \leq \omega$. We may endow these with the $C^{r}$ topology (for Homeo $\mathrm{H}_{+}\left(S^{1}\right), r=0$ ) and study the function $\rho$ by investigating subspaces on which $\rho$ takes on certain values. For example, $\rho^{-1}(\mathbb{Q})$ contains an open and dense subset of $\mathrm{Homeo}_{+}\left(S^{1}\right)$, and $\rho^{-1}(\mathbb{Q}) \cap \operatorname{Diff}_{+}^{r}\left(S^{1}\right)$ contains an open and dense subset of $\operatorname{Diff}_{+}^{r}\left(S^{1}\right)$ for all $r \geq 1$ (see [4).

One can gain some insight into the local structure of $\rho$ by considering it on 1-parameter families of homeomorphisms of the circle. Whenever we have a 1parameter family of homeomorphisms $f_{t}$, we will write $\rho(t):=\rho\left(f_{t}\right)$.

One way to get a 1-parameter family is to let $f$ be a homeomorphism and then define a family $f_{t}$ by $f_{t}=R_{t} \circ f$, where $R_{t}$ is rotation by $t$. The behavior of $\rho(t)$ is very different when its value is rational than when its value is irrational. For any $\alpha \notin \mathbb{Q}, \rho^{-1}(\alpha)$ will be a single point. This can be seen in the following way. Without loss of generality, $\rho(f)=\alpha$. It suffices to show that for arbitrarily small $t>0$ and arbitrarily small $t<0, f_{t}$ has periodic points.

Since $\rho(f)=\alpha \notin \mathbb{Q}$, either $f$ is conjugate to an irrational rotation, in which case every orbit is dense, or it is semi-conjugate to an irrational rotation, in which case there exists a unique invariant Cantor set on which the dynamics are minimal. In the first case, let $x \in S^{1}$ be arbitrary. Since the orbit of $x$ accumulates on $x$ on both sides and any finite subset of the orbit depends continuously on $t$, for arbitrarily small $t$ (both positive and negative) composing by $R_{t}$ will "close up" the orbit of $x$ so that $f_{t}$ will have periodic points. If $f$ is merely semi-conjugate to $R_{\alpha}$, let $x$ be in the invariant Cantor set. Choose it to be an accumulation point from the left if we want to find small $t>0$ such that $f_{t}$ has periodic points, and similarly for $t<0$. Once we find such an $x$, we argue as above.

On the other hand, for a generic (countable intersection of open and dense sets) $f \in \mathrm{Homeo}_{+}\left(S^{1}\right)$ or $\operatorname{Diff}_{+}^{r}\left(S^{1}\right)$ in the $C^{r}$ topology $(0 \leq r \leq \omega)$, for every $\frac{p}{q} \in \mathbb{Q}$, $\underline{\rho}^{-1}\left(\frac{p}{q}\right)$ is a nontrivial interval $([\underline{5}$, p. 37$)$.

The most famous example where $\underline{\rho}^{-1}\left(\frac{p}{q}\right)$ is a nontrivial interval for all $\frac{p}{q} \in \mathbb{Q}$, due to Arnold (as cited in Ghys [3, p. 358), is the following: $f_{a}(x)=x+a \cdot \sin (2 \pi x)$, where $a$ is chosen to be small enough so that $f$ is a homeomorphism. As above, $\left(f_{a}\right)_{t}$ is defined to be $R_{t} \circ f_{a}$. For $\frac{p}{q} \in \mathbb{Q}$, the set of $\left(t_{0}, a_{0}\right)$ in the $(t, a)$-plane such that $\rho\left(\left(f_{a_{0}}\right)_{t_{0}}\right)=\frac{p}{q}$ will be a strange "tongue" shape. These Arnold tongues are much studied and quite important.

In this paper, we consider a different type of 1-parameter family of circle homeomorphisms. We make the strong, rather unusual assumption that the rotation number is a strictly monotone function of the parameter; in particular, it has no "plateaus" when its value is rational, unlike in the above situations. We draw some strong conclusions in this context.

The first result says that in the special case where the rotation number is not just monotone but the identity, the behavior is highly constrained. 
Theorem 1. Suppose that $f: S^{1} \times S^{1} \rightarrow S^{1} \times S^{1}$ is $C^{2}$, with $f(x, t)=\left(f_{t}(x), t\right)$, and the rotation number of $f_{t}$ is equal to $t$ for all $t \in S^{1}$. Then for each $t$ there is a conjugacy $\phi_{t}$ from $f_{t}$ to $R_{t}$ (i.e. $f_{t}=\phi_{t}^{-1} \circ R_{t} \circ \phi_{t}$ ) such that $\phi_{t}$ varies continuously with $t$. In particular, $f$ is topologically conjugate to the linear Dehn twist of the torus $\left(\begin{array}{ll}1 & 1 \\ 0 & 1\end{array}\right)$.

We will see, in the course of the proof, that when $t \in \mathbb{Q}$ the conjugacy between $f_{t}$ and $R_{t}$ is actually forced to be differentiable.

We will show by way of example that the result of Theorem 1 does not hold in general if we only assume that the rotation number is strictly monotone. However, interesting properties continue to hold in that generality, which we explore in Theorem 2.

Note that $\frac{\partial f_{t}^{q}}{\partial t}\left(x, t_{0}\right)$ (or $\left.\frac{\partial f_{t}^{q}}{\partial t}\right|_{t=t_{0}}(x)$ if we wish to vary $x$ and hold $t$ constant) means $p r_{1}\left(\frac{\partial f^{q}}{\partial t}\left(x, t_{0}\right)\right)$, where $p r_{1}$ is a projection onto the first component.

Theorem 2. Suppose that $f: S^{1} \times[a, b] \rightarrow S^{1} \times[a, b]$ is $C^{2}$, with $f(x, t)=\left(f_{t}(x), t\right)$, and the rotation number is a strictly monotone increasing function of $t$. Let $t_{0}$ be such that $\underline{\rho}\left(t_{0}\right)=\frac{p}{q} \in \mathbb{Q}$ (where this fraction is reduced). Then $\underline{\rho}$ is differentiable at $t_{0}$. Moreover, if $\underline{\rho}^{\prime}\left(t_{0}\right) \neq 0$, then $\frac{\partial f_{t}^{q}}{\partial t}\left(x, t_{0}\right) \neq 0$ for all $x \in S^{1}$. In that case,

$$
\underline{\rho}^{\prime}\left(t_{0}\right)=\frac{1}{q \cdot \int_{S^{1}} \frac{1}{\frac{\partial f_{t}^{q}}{\partial t}\left(x, t_{0}\right)} d x} .
$$

As we will discuss below, this relates to classic results about the derivative of the rotation number when the value of the rotation number is irrational (see Herman, [4]). It stands in sharp contrast to how the rotation number behaves at rational values in families given by composing by rotation by $t$; see Matsumoto [6].

Remark 3. The arguments in this paper actually work in somewhat greater generality than we have assumed in Theorems 1 and 2. They imply the conclusion of Theorem 2 if we replace strict monotonicity of the rotation number with the following weaker condition:

$(\star)$ Whenever $\rho\left(t_{0}\right) \in \mathbb{Q}$, there exists $\epsilon>0$ such that either $\rho(t)>\rho\left(t_{0}\right)$ for $t \in\left(t_{0}, t_{0}+\epsilon\right)$ and $\underline{\rho}(t)<\underline{\rho}\left(t_{0}\right)$ for $t \in\left(t_{0}-\epsilon, t_{0}\right)$ or $\underline{\rho}(t)<\underline{\rho}\left(t_{0}\right)$ for $t \in\left(t_{0}, t_{0}+\epsilon\right)$ and $\underline{\rho}(t)>\underline{\rho}\left(t_{0}\right)$ for $t \in\left(t_{0}^{-}-\epsilon, t_{0}\right)$.

Similarly, in Theorem 1, instead of assuming $\rho(t)=t$, we could have assumed $\rho$ is strictly monotone and has nonzero derivative when its value is rational. To extend Theorem 1 still more, we can replace strictly monotone with $(\star)$, and we get conjugacy to the map of the torus that preserves the circles $C_{t}$ of constant $t$, acting on $C_{t}$ by $R_{\rho\left(f_{t}\right)}$. These generalizations of Theorem 1 rely on the fact that the rotation number is differentiable when its value is rational, even in the generality of $(\star)$, by Theorem 2 .

\section{Proof of TheOREM 1}

Throughout the proof, $f$ is as in the statement of Theorem 1 . We use $g$ or $g_{t}$ to refer to more general homeomorphisms or families of homeomorphisms of the circle.

Proof of Theorem 1. First suppose $t \notin \mathbb{Q}$. In that case, $f_{t}$ is $C^{2}$ and has irrational rotation number $t$, so by Denjoy's theorem, $f_{t}$ is conjugate to $R_{t}$. Moreover, since 
the only homeomorphisms of the circle that commute with a given irrational rotation are themselves rotations, if $\phi_{t}$ and $\tilde{\phi}_{t}$ are two conjugacies from $f_{t}$ to $R_{t}$, then they will differ by only a rotation. If we agree once and for all that the conjugacies should send 0 to 0 , then we have a well defined $\phi_{t}$ for each irrational $t$.

Now what we must show is that these $\phi_{t}(t \notin \mathbb{Q})$ vary continuously in $t$ and that they extend continuously to conjugacies for $t \in \mathbb{Q}$. We note that the assumption that $\rho(t)=t$, instead of merely being strictly monotone, becomes important in Lemma 7 , one of whose conditions is that the function $\underline{\rho}$ not have a derivative equal to 0 when its value is 0 .

The following lemma implies that the $\phi_{t}(t \notin \mathbb{Q})$ vary continuously in $t$.

Lemma 4. Let $g, g^{\prime}: S^{1} \rightarrow S^{1}$ be conjugate to irrational rotations, say $g=\phi^{-1} \circ$ $R_{\alpha} \circ \phi$ and $g^{\prime}=\left(\phi^{\prime}\right)^{-1} \circ R_{\alpha^{\prime}} \circ \phi^{\prime}$, where $\phi$ and $\phi^{\prime}$ are chosen to send 0 to 0 . For any $\epsilon>0$, there is a $\delta>0$ such that if $\left|g-g^{\prime}\right|_{C^{0}}<\delta$, then $\left|\phi-\phi^{\prime}\right|_{C^{0}}<\epsilon$.

Proof. Conjugating $g$ and $g^{\prime}$ by $\phi$, we may assume that $g=R_{\alpha}$. There is no loss of generality, since conjugation by $\phi$ is a continuous mapping of $\operatorname{Homeo}_{+}\left(S^{1}\right)$. Therefore, we have reduced to the case where $g=R_{\alpha}$, i.e. $\phi=i d$.

Let $\epsilon>0$. Choose $N$ large enough so that the points $0, \alpha, \ldots, N \alpha$ are $\epsilon$-dense in $S^{1}$ in the sense that the intervals of $S^{1} \backslash\{0, \ldots, N \alpha\}$ all have length less than $\epsilon$. If $g^{\prime}$ is sufficiently close to $R_{\alpha}$, we will have $\left|\alpha-\alpha^{\prime}\right|<\frac{\epsilon}{N}$, which implies that the intervals of $S^{1} \backslash\left\{0, \ldots, N \alpha^{\prime}\right\}$ have length less than $2 \epsilon$.

First we show that $\phi^{\prime}$ is close to the identity on the points $\left(g^{\prime}\right)^{n}(0), 0 \leq n \leq N$. By the choice of $\alpha^{\prime}$,

$$
\left|n \alpha-n \alpha^{\prime}\right|<\epsilon
$$

when $0 \leq n \leq N$. If $g^{\prime}$ is close enough to $R_{\alpha}$, then we will have

$$
\left|n \alpha-\left(g^{\prime}\right)^{n}(0)\right|<\epsilon
$$

for all $0 \leq n \leq N$. Therefore,

$$
\left|\left(g^{\prime}\right)^{n}(0)-n \alpha^{\prime}\right|<2 \epsilon .
$$

Now we show that $\phi^{\prime}$ is close to the identity everywhere. Let $x \in S^{1}$, and let $0 \leq m, n \leq N$ be such that $\left(\left(g^{\prime}\right)^{m}(0),\left(g^{\prime}\right)^{n}(0)\right)$ is one of the intervals of $S^{1} \backslash\left\{0, \ldots,\left(g^{\prime}\right)^{N}(0)\right\}$ and $x \in\left[\left(g^{\prime}\right)^{m}(0),\left(g^{\prime}\right)^{n}(0)\right]$. Then $m \alpha^{\prime} \leq \phi^{\prime}(x) \leq n \alpha^{\prime}$. Since $\left(g^{\prime}\right)^{m}(0)$ is within $2 \epsilon$ of $m \alpha^{\prime}$ and $\left(g^{\prime}\right)^{n}(0)$ is within $2 \epsilon$ of $n \alpha^{\prime}$,

$$
\left|\phi^{\prime}(x)-x\right|<2 \epsilon+(n-m) \alpha^{\prime} .
$$

Recall that $(n-m) \alpha^{\prime}<2 \epsilon$, so

$$
\left|\phi^{\prime}(x)-x\right|<4 \epsilon \text {. }
$$

Since $\epsilon$ can be made arbitrarily small, we are done.

Remark 5. This reasoning also applies if $g^{\prime}$ is conjugate to a rational rotation. In that case, it says that for any $\epsilon>0$, there exists $\delta>0$ such that if $\left|g-g^{\prime}\right|_{C^{0}}<\delta$, then $\left|\phi-\phi^{\prime}\right|_{C^{0}}<\epsilon$, where $\phi^{\prime}$ is any conjugacy of $g^{\prime}$ to a rotation that sends 0 to 0 .

It is necessary to assume that $g$ is conjugate to an irrational rotation. The following statement is false: for any circle homeomorphism $g$ which is conjugate to a rotation, for any $\epsilon>0$, there exists a $\delta$ such that if $g^{\prime}$ is a circle homeomorphism conjugate to a rotation satisfying $\left|g-g^{\prime}\right|_{C^{0}}<\delta$, then there exist circle homeomorphisms $\phi$ and $\phi^{\prime}$ such that $g=\phi^{-1} \circ R_{\rho(g)} \circ \phi, g^{\prime}=\left(\phi^{\prime}\right)^{-1} \circ R_{\rho\left(g^{\prime}\right)} \circ \phi^{\prime}$, and $\left|\phi-\phi^{\prime}\right|_{C^{0}}<\epsilon$. 
We may construct a counterexample as follows. Let $\psi_{\epsilon}: S^{1} \rightarrow S^{1}$ be the homeomorphism which is linear on the intervals $\left[0, \frac{1}{4}\right),\left[\frac{1}{4}, \frac{1}{4}+\epsilon\right),\left[\frac{1}{4}+\epsilon, \frac{3}{4}-\epsilon\right),\left[\frac{3}{4}-\epsilon, \frac{3}{4}\right)$, and $\left[\frac{3}{4}, 1\right)$, with

$$
\begin{array}{ll}
\psi_{\epsilon}\left(\left[0, \frac{1}{4}\right)\right) & =\left[0, \frac{1}{8}\right), \\
\psi_{\epsilon}\left(\left[\frac{1}{4}, \frac{1}{4}+\epsilon\right)\right) & =\left[\frac{1}{8}, \frac{3}{8}\right), \\
\psi_{\epsilon}\left(\left[\frac{1}{4}+\epsilon, \frac{3}{4}-\epsilon\right)\right) & =\left[\frac{3}{8}, \frac{5}{8}\right), \\
\psi_{\epsilon}\left(\left[\frac{3}{4}-\epsilon, \frac{3}{4}\right)\right) & =\left[\frac{5}{8}, \frac{7}{8}\right), \\
\psi_{\epsilon}\left(\left[\frac{3}{4}, 1\right)\right) & =\left[\frac{7}{8}, 1\right) .
\end{array}
$$

For $\epsilon$ sufficiently small, we will have $\psi_{\epsilon}^{-1} \circ R_{\frac{1}{2}} \circ \psi_{\epsilon} \approx R_{\frac{1}{2}}$. To see this, consider $\psi(x)=\lim _{\epsilon \rightarrow 0} \psi_{\epsilon}^{-1}(x) . \psi$ is no longer a homeomorphism: it will send $\left[\frac{1}{8}, \frac{3}{8}\right]$ to $\left\{\frac{1}{4}\right\}$ and $\left[\frac{5}{8}, \frac{7}{8}\right]$ to $\left\{\frac{3}{4}\right\}$. Nonetheless, we have $\psi \circ R_{\frac{1}{2}}=R_{\frac{1}{2}} \circ \psi$.

Even though $\psi_{\epsilon}^{-1} \circ R_{\frac{1}{2}} \circ \psi_{\epsilon} \approx R_{\frac{1}{2}}, \psi_{\epsilon}$ is not close to any homeomorphism that commutes with $R_{\frac{1}{2}}$, since $\psi_{\epsilon} \circ R_{\frac{1}{2}}\left(\frac{1}{4}\right)=\frac{7}{8}$, which is far from $R_{\frac{1}{2}} \circ \psi_{\epsilon}\left(\frac{1}{4}\right)=\frac{5}{8}$.

Let $g=R_{\frac{1}{2}}$. Let $\epsilon$ be small enough that $\psi_{\epsilon}^{-1} \circ R_{\frac{1}{2}} \circ \psi_{\epsilon}$ is close to $R_{\frac{1}{2}}$, and let $\alpha^{\prime} \notin \mathbb{Q}$ be close enough to $\frac{1}{2}$ that $g^{\prime}=\psi_{\epsilon}^{-1} \circ R_{\alpha^{\prime}} \circ \psi_{\epsilon}$ is still close to $R_{\frac{1}{2}}$. Despite the fact that $g^{\prime}$ is arbitrarily close to $g$, no conjugacy for $g^{\prime}$ is close to any conjugacy for $g$ (since $g^{\prime}$ is conjugate to an irrational rotation, $\psi_{\epsilon}$ is essentially the only conjugacy for $\left.g^{\prime}\right)$.

The following lemma shows that $f_{0}=i d$.

Lemma 6. If a homeomorphism $g$ of the circle has $\rho(g)=0$ and there exist homeomorphisms arbitrarily close to $g$ with a positive rotation number, as well as homeomorphisms arbitrarily close with a negative rotation number, then $g=i d$.

Proof. By assumption, $\rho(g)=0$, so $g$ has fixed points. The intuition of the lemma is as follows. If $g \neq i d$, then either its graph crosses the diagonal $y=x$ or does not cross but is tangent to the diagonal at some points.

If the graph crosses the diagonal, then under slight perturbations it will still have fixed points, hence still have rotation number 0 . If it is tangent to the diagonal but does not cross, then under small perturbations we may increase or decrease (but not both) the rotation number. The reason is that in this situation the graph either does not go below or does not go above the diagonal. Suppose it does not go below the diagonal. Then by pushing the graph upward slightly we make the rotation number positive, but if we push it downward the rotation number will still be 0 . Similarly, if the graph does not go above the diagonal, then by applying a small perturbation, we may decrease, but not increase, the rotation number. The details are left as an exercise for the reader.

The following lemma implies that $\frac{\partial f_{t}}{\partial t}\left(x, t_{0}\right)$ is greater than 0 for all $x$, since $\underline{\rho}^{\prime}(0)=1$.

Lemma 7. Suppose $g: S^{1} \times[a, b] \rightarrow S^{1} \times[a, b]$ is $C^{2}$, with $g(x, t)=\left(g_{t}(x), t\right)$, and the rotation number is a strictly monotone increasing function of $t$, with $\rho\left(t_{0}\right)=0$. Suppose it is not the case that the derivative $\underline{\rho}^{\prime}\left(t_{0}\right)$ exists and is equal to 0 . Then $\frac{\partial g_{t}}{\partial t}\left(x, t_{0}\right)$ is greater than 0 for all $x$. 
Proof. We must show that the function $\left.\frac{\partial g_{t}}{\partial t}\right|_{t=t_{0}}(x)$ is strictly greater than 0 . By Lemma 6. $g_{t_{0}}=i d$. For any $t>t_{0}, g_{t}(x)>x$ for all $x$, and similarly for $t<t_{0}$; this must be true because $\rho$ is strictly monotone increasing. Therefore, for any $x$, $\left.\frac{\partial g_{t}}{\partial t}\right|_{t=t_{0}}(x) \geq 0$. It remains to show that $\left.\frac{\partial g_{t}}{\partial t}\right|_{t=t_{0}}(x) \geq 0$ never vanishes.

Suppose, by way of contradiction, that for some $x_{0},\left.\frac{\partial g_{t}}{\partial t}\right|_{t=t_{0}}\left(x_{0}\right)=0$. Since the function $\left.\frac{\partial g_{t}}{\partial t}\right|_{t=t_{0}}$ is nonnegative and $C^{1}$ (because $g$ is $C^{2}$ ), it will have a minimum at $x_{0}$. Therefore, for any $\epsilon>0$ there exists $\delta>0$ such that when $\left|x-x_{0}\right|<\delta$, $\left.\frac{\partial g_{t}}{\partial t}\right|_{t=t_{0}}(x)<\epsilon\left|x-x_{0}\right|$.

Since it is not the case that $\rho^{\prime}\left(t_{0}\right)$ exists and is equal to 0 and $\rho$ is monotone increasing, there exist $\eta>0$ and $t$ arbitrarily close to $t_{0}$ such that $\frac{\underline{\rho}(t)-\underline{\rho}\left(t_{0}\right)}{t-t_{0}}>\eta$. Without loss of generality there are $t>t_{0}$ arbitrarily close to $t_{0}$ with this property. We may rewrite it as $\underline{\rho}(t)>\eta\left(t-t_{0}\right)$. Let $Q=\frac{2}{\eta}+3\left(t-t_{0}\right)$, and choose a $\delta$ that works for $\epsilon=\frac{1}{Q}$. For $t$ sufficiently close to $t_{0}$, we have that for all $x$,

$$
g_{t}(x)-x \in\left(\left(\left.\frac{\partial g_{t}}{\partial t}\right|_{t=t_{0}}(x)-\frac{\delta}{Q}\right)\left(t-t_{0}\right),\left(\left.\frac{\partial g_{t}}{\partial t}\right|_{t=t_{0}}(x)+\frac{\delta}{Q}\right)\left(t-t_{0}\right)\right) .
$$

Fix a $t>t_{0}$ which is this close to $t_{0}$ and which has the property that $\underline{\rho}(t)>\eta\left(t-t_{0}\right)$.

Consider what iterates of $g_{t}$ do to $x_{0}$. We have

$$
g_{t}\left(x_{0}\right)-x_{0}<\frac{\delta}{Q}\left(t-t_{0}\right) .
$$

Since this is less than $\delta$,

$$
g_{t}^{2}\left(x_{0}\right)-x_{0}<\left(\frac{\delta}{Q}+\frac{2 \delta}{Q}\right)\left(t-t_{0}\right)=\frac{3 \delta}{Q}\left(t-t_{0}\right) .
$$

This pattern will continue; in general, we will have

$$
g_{t}^{n}\left(x_{0}\right)-x_{0}<\frac{2 n-1}{Q} \delta\left(t-t_{0}\right)
$$

as long as $\frac{2 n-1}{Q} \delta\left(t-t_{0}\right)<\delta$. This says that when $\frac{2 n-1}{Q} \delta\left(t-t_{0}\right)<\delta$, i.e. when $n<\frac{Q}{2\left(t-t_{0}\right)}+\frac{1}{2}$, or (substituting in the value $Q=\frac{2}{\eta}+3\left(t-t_{0}\right)$ ) when $n<\frac{1}{\eta\left(t-t_{0}\right)}+2$, then

$$
g_{t}^{n}\left(x_{0}\right)-x_{0}<\delta
$$

Now notice that since $\underline{\rho}(t)>\eta\left(t-t_{0}\right)$, there is an integer $n \leq \frac{1}{\eta\left(t-t_{0}\right)}+1$ such that after applying $g_{t} n$ times starting at $x_{0}$, we will have made a full circle. But equation (2) says that after applying $g_{t}$ this many times we will not have moved farther than $\delta$; this is a contradiction.

In the following lemma, note that $t$ plays two roles: in $\phi_{t}$ and as a dummy variable in $\frac{\partial f_{t}}{\partial t}(s, 0)$.

Lemma 8. As $t \rightarrow 0(t \notin \mathbb{Q})$, the conjugacies $\phi_{t}$ approach the function $x \mapsto$ $\int_{0}^{x} \frac{1}{\frac{\partial f_{t}}{\partial t}(s, 0)} d s$. Therefore, the conjugacies extend continuously to $t=0$.

Proof. We must show that for every $x, \phi_{t}(x) \rightarrow \int_{0}^{x} \frac{1}{\frac{\partial f_{t}}{\partial t}(s, 0)} d s$ as $t \rightarrow 0$. 
Choose $t$ small enough so that for any $x \in S^{1}$,

$$
\int_{x}^{f_{t}(x)} \frac{1}{\frac{\partial f_{t}}{\partial t}(s, 0)} d s \in((1-\epsilon) t,(1+\epsilon) t) .
$$

Let $n$ be such that $n t \leq \phi_{t}(x) \leq(n+1) t$. Note that

$$
\int_{0}^{x} \frac{1}{\frac{\partial f_{t}}{\partial t}(s, 0)} d s \approx \int_{0}^{f_{t}^{n}(0)} \frac{1}{\frac{\partial f_{t}}{\partial t}(s, 0)} d s
$$

and the latter integral can be broken into integrals of the form $\int_{f_{t}^{i}(0)}^{f_{t}^{i+1}(0)} \frac{1}{\frac{\partial f_{t}}{\partial t}(s, 0)} d s$, each of which lies in $((1-\epsilon) t,(1+\epsilon) t)$. In this way, we find that

$$
\frac{1}{(1+\epsilon) t} \int_{0}^{x} \frac{1}{\frac{\partial f_{t}}{\partial t}(s, 0)} d s-1<n<\frac{1}{(1-\epsilon) t} \int_{0}^{x} \frac{1}{\frac{\partial f_{t}}{\partial t}(s, 0)} d s .
$$

Now note that $\phi_{t}(x)$ lies between $n t$ and $(n+1) t$, so it differs from $n t$ by at most t. Also, $n t$ satisfies

$$
\frac{1}{1+\epsilon} \int_{0}^{x} \frac{1}{\frac{\partial f_{t}}{\partial t}(s, 0)} d s-t<n t<\frac{1}{1-\epsilon} \int_{0}^{x} \frac{1}{\frac{\partial f_{t}}{\partial t}(s, 0)} d s .
$$

When $t$ and $\epsilon$ go to 0 , the difference between $n t$ and $\int_{0}^{x} \frac{1}{\frac{\partial f_{t}}{\partial t}(s, 0)} d s$, and hence also the difference between $\phi_{t}(x)$ and $\int_{0}^{x} \frac{1}{\frac{\partial f_{t}}{\partial t}(s, 0)} d s$, goes to 0 .

To finish the proof of Theorem 1, observe that there's nothing special about $t=0$ as opposed to any other rational number. For any $t=\frac{p}{q}$ (a reduced fraction), we may consider the function $f^{q}$. By Lemma 6, this is the identity on the circle $\left\{t=\frac{p}{q}\right\}$, and nearby it will look just like $f$ did near $t=0$. In particular, we can look at $\left.\frac{\partial f_{t}^{q}}{\partial t}\right|_{t=\frac{p}{q}}$; this will be strictly greater than 0 , and we will get a function that the conjugacies must approach as $t \rightarrow \frac{p}{q}, t \notin \mathbb{Q}$. (Note that for $t \notin \mathbb{Q}$, the conjugacy $\phi_{t}$ from $f_{t}$ to $R_{t}$ is also the conjugacy from $f_{t}^{q}$ to $R_{q t}$.)

Therefore, we have a family of conjugacies $\phi_{t}$ defined for every $t$ which is continuous in $t$, as desired.

We note that the above result does not hold if we merely require the rotation number to be strictly monotone in $t$.

Example. Define a family $f_{t}$ parametrized by $t \in S^{1}$ by

$$
f_{t}=\left\{\begin{array}{cc}
i d, & t=0, \\
\phi_{t}^{-1} \circ R_{\underline{\rho}(t)} \circ \phi_{t}, & t \neq 0,
\end{array}\right.
$$

where $\underline{\rho}: S^{1} \rightarrow S^{1}$ is $1-1$, sends 0 to 0 , and is "exponentially flat" about 0 (i.e. there exists $\epsilon>0$ such that for all $\left.|t|<\epsilon,|\underline{\rho}(t)|<e^{-\frac{1}{|t|}}\right)$, and $\phi_{t}$ is defined to be

$$
\phi_{t}(x)=x+\frac{1}{4 \pi} \sin \left(\frac{1}{t}\right) \sin (2 \pi x)
$$

for $t \neq 0$. 
Due to the exponential flatness of $\rho$, the family $f_{t}$ is $C^{\infty}$. The given conjugacies do not vary continuously as $t \rightarrow 0$, so it looks as if $f$ may not be topologically conjugate to the map $(x, t) \mapsto\left(R_{\rho(t)}(x), t\right)$, which itself is conjugate to the map $\left(\begin{array}{ll}1 & 1 \\ 0 & 1\end{array}\right)$ of the torus. Let us see why this is true: $f$ is not conjugate to $\left(\begin{array}{ll}1 & 1 \\ 0 & 1\end{array}\right)$.

Let us denote by $C_{t}$ the circle $\left\{(x, t): x \in S^{1}\right\}$; we will call these "horizontal circles". Suppose, by way of contradiction, that $\psi$ is a conjugacy such that $f=$ $\psi^{-1} \circ\left(\begin{array}{ll}1 & 1 \\ 0 & 1\end{array}\right) \circ \psi$. Then there are two possibilities: $\psi$ sends $C_{t}$ to $C_{\rho(t)}$ or $C_{-\rho(t)}$. The reason is the following. The torus is foliated by horizontal circles on which $f$ acts. The circles on which the rotation number is irrational are dense in this foliation, so where they are sent determines the conjugacy. Let $t$ be such that $\rho(t) \notin \mathbb{Q}$, and consider where $(0, t)$ is sent by $\psi$. If $\psi(0, t) \in C_{u}$, then the whole $\bar{f}_{t}$-orbit of $(0, t)$ must be sent into $C_{u}$, since $\left(\begin{array}{ll}1 & 1 \\ 0 & 1\end{array}\right)$ preserves horizontal circles. Since the orbit of $(0, t)$ is dense in $C_{t}$, the image of $C_{t}$ is $C_{u}$. The dynamics of $f$ on $C_{t}$ must be conjugate to the dynamics of $\left(\begin{array}{ll}1 & 1 \\ 0 & 1\end{array}\right)$ on $C_{u}$. This implies that $u=\rho(t)$ or $-\rho(t)$.

Suppose $\psi$ sends every $C_{t}$ to $C_{\rho(t)}$. When $\underline{\rho}(t) \notin \mathbb{Q}$, we know how $\psi$ maps $C_{t}$ to $C_{\underline{\rho}(t)}$ : up to a rotation, $\psi_{t}$ agrees with $\phi_{t}$ (since rotations are the only maps that commute with an irrational rotation). Define a function $k(t)=\psi_{t}(0)$; note that, since $\psi$ is assumed to be continuous, $k$ is continuous. When $\underline{\rho}(t) \notin \mathbb{Q}$, we have $\psi_{t}(x)=k(t)+\phi_{t}(x)$. In fact, even if $\rho\left(t_{0}\right) \in \mathbb{Q}\left(\rho\left(t_{0}\right) \neq 0\right)$, we can choose $t \notin \mathbb{Q} \rightarrow t_{0}$. As $t \rightarrow t_{0}, k(t) \rightarrow k\left(t_{0}\right)$ and $\bar{\phi}_{t} \rightarrow \phi_{t_{0}}$, so $\psi_{t} \rightarrow k\left(t_{0}\right)+\phi_{t_{0}}=\psi_{t_{0}}$. Therefore, for all $x$ and $t \neq 0$,

$$
\psi_{t}(x)=k(t)+\phi_{t}(x) .
$$

But notice that

$$
\psi_{t}\left(\frac{1}{4}\right)=k(t)+\frac{1}{4}+\frac{1}{4 \pi} \sin \left(\frac{1}{t}\right) .
$$

Since $k(t)$ approaches a limit as $t \rightarrow 0$ and $\frac{1}{4 \pi} \sin \left(\frac{1}{t}\right)$ does not, $\psi_{t}\left(\frac{1}{4}\right)$ does not approach a limit as $t \rightarrow 0$. Therefore, $\psi$ is not continuous, a contradiction.

Now suppose that $\psi$ sends $C_{t}$ to $C_{-\rho(t)}$. Arguing as above, for some continuous function $k(t)$ we will have $\psi_{t}(x)=k(t)-\phi_{t}(x)$ for all $x$ and $t \neq 0$. But as above, if we plug in $x=\frac{1}{4}$ and let $t \rightarrow 0, \psi_{t}\left(\frac{1}{4}\right)$ does not approach a limit, contradicting the assumption that $\psi$ is continuous.

Therefore, we have a $C^{\infty}$ family of circle diffeomorphisms parametrized by $t \in S^{1}$ with rotation number a strictly monotone increasing injective function $S^{1} \rightarrow S^{1}$, conjugate at each $t$ to a rotation by $\underline{\rho}(t)$ but not globally conjugate to $\left(\begin{array}{ll}1 & 1 \\ 0 & 1\end{array}\right)$.

\section{Proof of Theorem 2}

Before we prove Theorem 2, we remark that the subject of the derivative of the rotation number in 1-parameter families has a long and rich history. In [4, Herman essentially showed that the function $\rho$ : $\operatorname{Diff}_{+}^{1}\left(S^{1}\right) \rightarrow \mathbb{R} / \mathbb{Z}$ is (Fréchet) differentiable at irrational rotations. The derivative is the map

$$
\phi \in C^{1}\left(S^{1}\right) \mapsto \int_{S^{1}} \phi(x) d x \in \mathbb{R} .
$$

He used this to derive a result originally due to Brunovský [1] that if $f_{t}$ is a family of homeomorphisms of the circle varying in a $C^{1}$ way, and if $f_{0}$ is an irrational rotation, then $\underline{\rho}(t)$ is differentiable at $t=0$, with derivative $\int_{S^{1}} \frac{\partial f_{t}}{\partial t}(x, 0) d x$. As 
a corollary, he showed that if $f_{0}$ is conjugate to an irrational rotation, say $f_{0}=$ $\phi^{-1} \circ R_{\alpha} \circ \phi$, then $\underline{\rho}(t)$ is still differentiable at $t=0$, with derivative

$$
\underline{\rho}^{\prime}(0)=\int_{S^{1}} \phi^{\prime}\left(f_{0}\left(\phi^{-1}(x)\right)\right) \cdot \frac{\partial f_{t}}{\partial t}\left(\phi^{-1}(x), 0\right) d x
$$

(his notation was slightly different).

Recently, Matsumoto 6] showed the following: Let $f$ be a real-analytic diffeomorphism of the circle and let $f_{t}=R_{t} \circ f$. Suppose $\underline{\rho}(t)=\frac{p}{q}$ and $t$ is an endpoint of a nontrivial interval on which $\underline{\rho}$ is constantly equal to $\frac{p}{q}$ (recall that for a generic $f \in \operatorname{Diff}_{+}^{\omega}\left(S^{1}\right), \underline{\rho}^{-1}\left(\frac{p}{q}\right)$ will be a nontrivial interval for all $\left.\frac{p}{q} \in \mathbb{Q}\right)$. Then

$$
\limsup _{t^{\prime} \rightarrow t} \frac{\underline{\rho}\left(t^{\prime}\right)-\underline{\rho}(t)}{t^{\prime}-t}=\infty .
$$

The hypotheses of Theorem 2 rule out this kind of behavior.

Proof of Theorem 2. We begin by supposing that $\rho\left(t_{0}\right)=0$; the general case of $\underline{\rho}\left(t_{0}\right)=\frac{p}{q}$ is not much harder. If $\underline{\rho}^{\prime}\left(t_{0}\right)=0$, then there is nothing to show. Otherwise, a priori either $\underline{\rho}^{\prime}\left(t_{0}\right)$ exists and is nonzero or it does not exist. We must show that only the first possibility can happen and the value of the derivative is given by the formula in the theorem.

By Lemma $7 \frac{\partial f_{t}}{\partial t}\left(x, t_{0}\right)$ is greater than 0 for all $x$. We want to show that

$$
\lim _{t \rightarrow t_{0}} \frac{\underline{\rho}(t)}{t-t_{0}}=\frac{1}{\int_{S^{1}} \frac{1}{\frac{\partial f_{t}}{\partial t}\left(x, t_{0}\right)} d x} .
$$

We demonstrate this limit for $t$ approaching $t_{0}$ from above, with the case of $t$ approaching $t_{0}$ from below being similar.

We want to define a "rotation time" $T_{t}$, meaning basically how many times we have to apply $f_{t}$ before we make a full circle. Let $\tilde{f}_{t}$ be the lift of $f_{t}$ with translation number $\tau\left(\tilde{f}_{t}\right) \in(0,1)$. Define $T_{t}$ to be the smallest integer such that $\tilde{f}_{t}^{T_{t}}(0) \geq 1$.

To show that

$$
\lim _{t \downarrow t_{0}} \frac{\rho(t)}{t-t_{0}}=\frac{1}{\int_{S^{1}} \frac{1}{\frac{\partial f_{t}}{\partial t}\left(x, t_{0}\right)} d x},
$$

it is equivalent to show that

$$
\lim _{t \downarrow t_{0}} \frac{t-t_{0}}{\underline{\rho}(t)}=\int_{S^{1}} \frac{1}{\frac{\partial f_{t}}{\partial t}\left(x, t_{0}\right)} d x .
$$

Notice that $T_{t} \in\left[\frac{1}{\underline{\rho}(t)}, \frac{1}{\underline{\rho}(t)}+1\right]$, so

$$
\lim _{t \downarrow t_{0}} \frac{t-t_{0}}{\underline{\rho}(t)}=\lim _{t \downarrow t_{0}}\left(t-t_{0}\right) T_{t}
$$

(provided either of these limits exists), and it suffices to show that

$$
\lim _{t \downarrow t_{0}}\left(t-t_{0}\right) T_{t}=\int_{S^{1}} \frac{1}{\frac{\partial f_{t}}{\partial t}\left(x, t_{0}\right)} d x .
$$


We will use the notation $\left.\frac{\partial f_{t}}{\partial t}\right|_{t=t_{0}}(x)$ for $\frac{\partial f_{t}}{\partial t}\left(x, t_{0}\right)$ to emphasize that $x$ is being varied. Let us consider Riemann sums for the integral $\int_{S^{1}} \frac{1}{\left.\frac{\partial f_{t}}{\partial t}\right|_{t=t_{0}}(x)} d x$. Specifically, let

$$
S_{N}=\sum_{n=0}^{N-1} \frac{1}{\left.\frac{\partial f_{t}}{\partial t}\right|_{t=t_{0}}\left(\frac{n}{N}\right)} \cdot \frac{1}{N}
$$

Since $\left.\frac{\partial f_{t}}{\partial t}\right|_{t=t_{0}}$ is strictly greater than 0 and continuous, $\frac{1}{\left.\frac{\partial f_{t}}{\partial t}\right|_{t=t_{0}}}$ is continuous, so $S_{N} \rightarrow \int_{S^{1}} \frac{1}{\left.\frac{\partial f_{t}}{\partial t}\right|_{t=t_{0}}(x)} d x$ as $N \rightarrow \infty$. It suffices to show that for sufficiently large $N$ and $t$ close enough to $t_{0},\left(t-t_{0}\right) T_{t}$ is within $\epsilon$ of $S_{N}$, since (if $N$ is large enough) $S_{N}$ will be within $\epsilon$ of $\int_{S^{1}} \frac{1}{\left.\frac{\partial f_{t}}{\partial t}\right|_{t=t_{0}}(x)} d x$.

For any $\eta>0$, for sufficiently large $N$, if we divide the circle into $N$ equal intervals $I_{0}=\left[0, \frac{1}{N}\right), \ldots, I_{N-1}=\left[\frac{N-1}{N}, 1\right)$, then for any $x \in I_{n}$,

$$
\left.\frac{\partial f_{t}}{\partial t}\right|_{t=t_{0}}(x) \in\left(\left.\frac{\partial f_{t}}{\partial t}\right|_{t=t_{0}}\left(\frac{n}{N}\right)-\eta,\left.\frac{\partial f_{t}}{\partial t}\right|_{t=t_{0}}\left(\frac{n}{N}\right)+\eta\right) .
$$

Furthermore, for any $\theta>0$ we can choose $t$ close enough to $t_{0}$ so that for all $x$,

$$
f_{t}(x)-x \in\left(\left(\left.\frac{\partial f_{t}}{\partial t}\right|_{t=t_{0}}(x)-\theta\right)\left(t-t_{0}\right),\left(\left.\frac{\partial f_{t}}{\partial t}\right|_{t=t_{0}}(x)+\theta\right)\left(t-t_{0}\right)\right) .
$$

Let us consider the orbit of 0 under $f_{t}$. We would like estimates on the number of iterates contained in each $I_{n}$; let us call this $\left(T_{t}\right)_{n}$. We have

$$
\left(T_{t}\right)_{n} \leq \frac{1}{\left(\left.\frac{\partial f_{t}}{\partial t}\right|_{t=t_{0}}\left(\frac{n}{N}\right)-\eta-\theta\right)\left(t-t_{0}\right) N} .
$$

Further, for any $\iota>0$ we can choose $t$ small enough so that

$$
\frac{1-\iota}{\left(\left.\frac{\partial f_{t}}{\partial t}\right|_{t=t_{0}}\left(\frac{n}{N}\right)+\eta+\theta\right)\left(t-t_{0}\right) N} \leq\left(T_{t}\right)_{n} .
$$

The number $\iota$ comes in because we have to consider how far to the right of $\frac{n}{N}$ the first iterate in $I_{n}$ is. Multiplying everything by $t-t_{0}$, we get

$$
\frac{1-\iota}{\left(\left.\frac{\partial f_{t}}{\partial t}\right|_{t=t_{0}}\left(\frac{n}{N}\right)+\eta+\theta\right) N} \leq\left(t-t_{0}\right)\left(T_{t}\right)_{n} \leq \frac{1}{\left(\left.\frac{\partial f_{t}}{\partial t}\right|_{t=t_{0}}\left(\frac{n}{N}\right)-\eta-\theta\right) N} .
$$

For any $\kappa>0$, we can therefore say that

$$
\left(t-t_{0}\right)\left(T_{t}\right)_{n} \in\left((1-\kappa) \frac{1}{\left.\frac{\partial f_{t}}{\partial t}\right|_{t=t_{0}}\left(\frac{n}{N}\right)} \cdot \frac{1}{N},(1+\kappa) \frac{1}{\left.\frac{\partial f_{t}}{\partial t}\right|_{t=t_{0}}\left(\frac{n}{N}\right)} \cdot \frac{1}{N}\right),
$$

provided we made $\eta, \theta$, and $\iota$ small enough. This says that

$$
\left(t-t_{0}\right) T_{t}=\left(t-t_{0}\right)\left(T_{t}\right)_{0}+\ldots+\left(t-t_{0}\right)\left(T_{t}\right)_{N-1} \in\left((1-\kappa) S_{N},(1+\kappa) S_{N}\right),
$$

and since $S_{N}$ is bounded above (independent of $N$ ), for sufficiently large $N$ and $t$ close enough to $t_{0}$ we have $\left(t-t_{0}\right) T_{t} \in\left(S_{N}-\epsilon, S_{N}+\epsilon\right)$. 
Finally, we must address the case where $\underline{\rho}\left(t_{0}\right)=\frac{p}{q} \neq 0$ (assume this fraction is reduced). Then we consider the family $g_{t}$, where $g_{t}=f_{t}^{q}$ for all $t$. Then $g_{t_{0}}$ has rotation number 0 . The above reasoning applied to the family $g_{t}$ says that

$$
\left.\frac{d}{d t}\right|_{t=t_{0}} \rho\left(g_{t}\right)=\frac{1}{\int_{S^{1}} \frac{1}{\frac{\partial g_{t}}{\partial t}\left(x, t_{0}\right)} d x} .
$$

But $\frac{\partial g_{t}}{\partial t}\left(x, t_{0}\right)=\frac{\partial f_{t}^{q}}{\partial t}\left(x, t_{0}\right)$ and $\left.\frac{d}{d t}\right|_{t=t_{0}} \rho\left(g_{t}\right)=\left.q \cdot \frac{d}{d t}\right|_{t=t_{0}} \rho\left(f_{t}\right)$, yielding the desired formula.

\section{REFERENCES}

[1] Pavol Brunovský, Generic properties of the rotation number of one-parameter diffeomorphisms of the circle, Czechoslovak Math. J. 24(99) (1974), 74-90. MR0346845 (49 \#11567)

[2] Welington de Melo and Sebastian van Strien, One-dimensional dynamics, Ergebnisse der Mathematik und ihrer Grenzgebiete (3) [Results in Mathematics and Related Areas (3)], vol. 25, Springer-Verlag, Berlin, 1993. MR,1239171 (95a:58035)

[3] Étienne Ghys, Groups acting on the circle, Enseign. Math. (2) 47 (2001), no. 3-4, 329-407. MR.1876932 (2003a:37032)

[4] Michael-Robert Herman, Mesure de Lebesgue et nombre de rotation, Geometry and topology (Proc. III Latin Amer. School of Math., Inst. Mat. Pura Aplicada CNPq, Rio de Janeiro, 1976), Lecture Notes in Math., Vol. 597, Springer, Berlin, 1977, pp. 271-293. MR0458480 (56 \#16682)

[5] Michael-Robert Herman, Sur la conjugaison différentiable des difféomorphismes du cercle à des rotations, Inst. Hautes Études Sci. Publ. Math. 49 (1979), 5-233 (French). MR538680 (81h:58039)

[6] Shigenori Matsumoto, Derivatives of rotation number of one parameter families of circle diffeomorphisms, Kodai Math. J. 35 (2012), no. 1, 115-125, DOI 10.2996/kmj/1333027257. MR 2911269

[7] Andrés Navas, Groups of circle diffeomorphisms, Translation of the 2007 Spanish edition, Chicago Lectures in Mathematics, University of Chicago Press, Chicago, IL, 2011. MR.2809110

Department of Mathematics, Northwestern University, 2033 Sheridan Road, EvansTON, ILLINOIS 60208-2730 